\title{
Magnetohydrodynamics flow of a nanofluid driven by a stretching/shrinking sheet with suction
}

\author{
U. S. Mahabaleshwar ${ }^{\text {* }}$, P. N. Vinay Kumar ${ }^{2}$ and Mikhail Sheremet ${ }^{3}$
}

*Correspondence: ulavathi@
gmail.com
${ }^{1}$ Department
of Mathematics, Government
First Grade College
for Women, Hassan,
Karnataka 573 201, India
Full list of author information
is available at the end of the
article

*Correspondence: ulavathi@ gmail.com

'Department

First Grade College

, Women, Hassan,

\begin{abstract}
The present paper investigates the effect of a mathematical model describing the aforementioned process in which the ambient nanofluid in the presence of suction/ injection and magnetic field are taken into consideration. The flow is induced by an infinite elastic sheet which is stretched along its own plane. The stretching/shrinking of the sheet is assumed to be proportional to the distance from the slit. The governing equations are reduced to a nonlinear ordinary differential equation by means of similarity transformation. The consequential nonlinear equation is solved analytically. Consequences show that the flow field can be divided into a near-field region and a far-field region. Suction on the surface plays an important role in the flow development in the near-field whereas the far-field is responsible mainly by stretching. The electromagnetic effect plays exactly the same role as the MHD, which is to reduce the horizontal flow resulting from stretching. It is shown that the behavior of the fluid flow changes with the change of the nanoparticles type. The present study throws light on the analytical solution of a class of laminar boundary layer equations arising in the stretching/shrinking sheet problem.
\end{abstract}

Keywords: Non-linear differential equation, Nanofluid, MHD stretching/shrinking sheet, Suction/injection

\section{Background}

Dynamics of fluid flow over a linear stretching/shrinking sheet plays very significant role in many manufacturing applications. The thin polymer sheet constitutes a continuously moving solid surface with a non-uniform surface velocity through an or else quiescent fluid. The cooling fluids in past times was selected to be the in large quantities available water, but this has the disadvantage of speedily quenching the heat leading to rapid solidification of the stretching sheet (see Andersson 1995, 2002, Fisher 1976, Siddheshwar and Mahabaleshwar 2005). From the standpoint of desirable properties of the final product water does not seem to be the ideal cooling fluid.

The word of nanofluid refers to a solid-liquid mixture with a continuous phase which is a nanometer sized nanoparticle dispersed in conventional base liquids. Nanofluids are base-fluids containing suspended nanoparticles. These nanoparticles are typically mad of metals, oxides, or carbon nanotubes. There are a few well-known correlations for predicting the thermal and physical properties of nanofluids which are often cited

(c) The Author(s) 2016. This article is distributed under the terms of the Creative Commons Attribution 4.0 International License (http://creativecommons.org/licenses/by/4.0/), which permits unrestricted use, distribution, and reproduction in any medium, provided you give appropriate credit to the original author(s) and the source, provide a link to the Creative Commons license, and indicate if changes were made. 

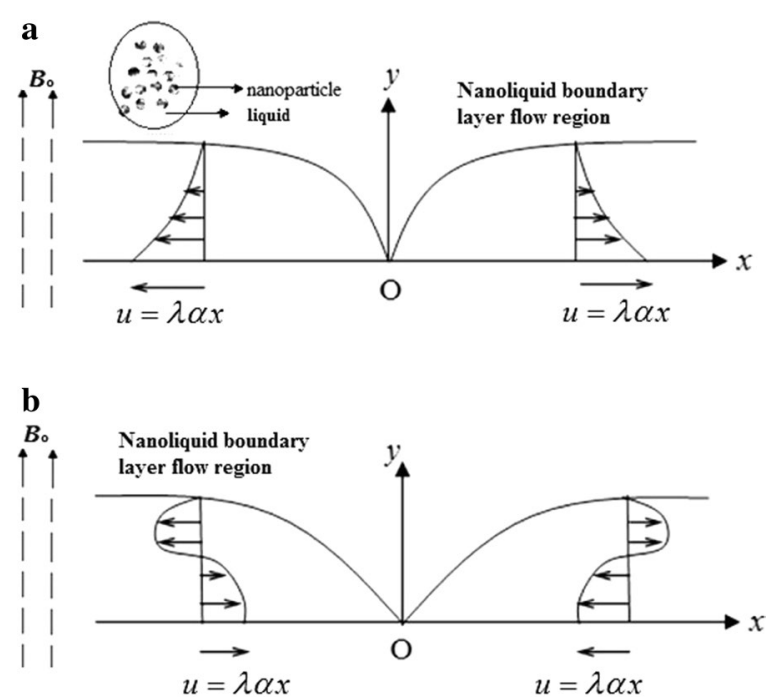

Fig. 1 The schematic flow diagram of stretching/shrinking boundary a Stretching sheet case $(\lambda>0)$ b Shrinking sheet case $(\lambda<0)$

by researchers to calculate the convective heat transfer behaviors of the nanofluids. The word "nanofluid" coined by Choi (1995) describes a liquid suspension containing ultrafine particles (diameter less than $50 \mathrm{~nm}$ ) (See Choi et al. 2001, Keblinski et al. 2005). The first providing for this ground of Sakiadis (1961a, b, c) he concentrated on the induced affected by the uniform motion of a continuous solid surface taking into account the laminar boundary layer approximation.

An exact analytical solution of the equation for a elastic sheet where the surface stretching velocity was proportional to the distance from the slot was given in Crane (1970). In this presentation, we will perform an analysis of a mathematical model describing the aforementioned process in which the ambient nanofluid in the presence of mass transfer is taken into consideration.

\section{Solution of mathematical formulation}

We reflect on the steady laminar boundary two-dimensional $(x, y)$ co-ordinate magnetohydrodynamic (MHD) flows of a nanofluid past stretching/shrinking sheet in the presence of presence of mass transfer. The liquid is electrically conducting in the presence of applied magnetic field with constant strength $B_{0}$ that is parallel to $y$-axis. The sheet is supposed extended in the $x$-direction such that the $x$-component of velocity varies linearly with $x$ along its surface. It is assumed that the velocity distribution of the stretching/shrinking sheet is $u=\lambda u_{w}(x)=\lambda \alpha x$ where $x$ is the coordinates calculated along the stretching/shrinking of the sheet, $\lambda$ is a constant with $\lambda>0$ for a stretching, $\lambda<0$ for a shrinking and $\lambda=0$ surface is permeable. Stretching/shrinking sheet problems Prandtl zero pressure gradient and outside other forces are not considered. In practice, it is only an extremely meticulous pulling of the sheet that can allow one to assume linear stretching. The schematic of the physical replica, geometrical coordinates are depicted in Fig. 1. The liquid is a based nanoliquids containing three types namely, $\mathrm{Cu}, \mathrm{Al}_{2} \mathrm{O}_{3}$ and $\mathrm{TiO}_{2}$. Thermophysical properties of the nanoliquid are listed in the below Table 1. 
Table 1 Thermophysical properties of the base fluid (water) and nanoparticles

\begin{tabular}{llccc}
\hline $\begin{array}{l}\text { Nanoliquid physical } \\
\text { properties }\end{array}$ & $\begin{array}{l}\text { Liquid phase } \\
\text { (water) }\end{array}$ & Copper & Alumina & Titania \\
\hline$C_{p}(\mathrm{~J} / \mathrm{kg} \mathrm{K})$ & 4179 & 385 & 765 & 686.2 \\
$\rho\left(\mathrm{kg} / \mathrm{m}^{3}\right)$ & 997.1 & 8933 & 3970 & 42.50 \\
$k(\mathrm{~W} / \mathrm{m} \mathrm{K})$ & 0.613 & 400 & 40 & 8.9538 \\
\hline
\end{tabular}

Conservation of mass and conservation linear momentum are given by

$$
\begin{aligned}
& \frac{\partial q_{i}}{\partial x_{i}}=0, \\
& \rho_{n f}\left[\frac{\partial q_{i}}{\partial t}+q_{j} \frac{\partial q_{i}}{\partial x_{j}}\right]=-\frac{\partial p}{\partial x_{i}}+\mu_{n f} \nabla^{2} q_{i}-\sigma B_{o}^{2} q_{i},
\end{aligned}
$$

where, $\frac{d}{d t}=\frac{\partial}{\partial t}+q_{j} \frac{\partial}{\partial x_{j}}$, where, the other quantities have their meaning as mentioned in nomenclature.

The bases for present analysis laminar boundary layer equations for an incompressible nanofluid.

$$
\begin{aligned}
& \frac{\partial u}{\partial x}+\frac{\partial v}{\partial y}=0, \\
& u \frac{\partial u}{\partial x}+v \frac{\partial u}{\partial y}=\frac{\mu_{n f}}{\rho_{n f}} \frac{\partial^{2} u}{\partial y^{2}}-\frac{\sigma B_{0}^{2}}{\rho_{n f}} u,
\end{aligned}
$$

where, the quantities have their meaning as mentioned in nomenclature. We further assume $R_{\mathrm{m}} \ll 1$, where $R_{\mathrm{m}}$ is the magnetic Reynolds number.

The associated boundary conditions on velocity are given by

$$
\begin{aligned}
& u=\lambda u_{w}(x)=\lambda \alpha x, \quad \mathrm{v}=\mathrm{v}_{c}, \quad \text { at } y=0, \\
& u \rightarrow 0, \quad \text { as } \quad y \rightarrow \infty .
\end{aligned}
$$

Laminar boundary layer flows induced by a continuous surface stretching with velocity $u_{w}(x), v_{c}$ is the mass flux velocity with $v_{c}<0$ for suction, $v_{c}>0$ for injection and $v_{c}=0$ is the case when the surface is impermeable.

In the physical stream function formulation $\psi(x, y)$ such that

$$
u=\frac{\partial \psi}{\partial y} \quad \text { and } \quad v=-\frac{\partial \psi}{\partial x},
$$

where, $\quad \psi(x, y)=\lambda \sqrt{v_{f}} x f(\eta), f(\eta)$ is the dimensionless stream function and $\eta=\left(\sqrt{\frac{\alpha}{v_{f}}}\right) y$. The material parameters in (4) are described mathematically by,

$$
\mu_{n f}=\frac{\mu_{f}}{(1-\phi)^{2.5}} \quad \text { and } \quad \rho_{n f}=\rho_{f}(1-\phi)+\rho_{s} \phi \text {, }
$$

where, $\phi(0<\phi<1)$ is the solid volume fraction, $\rho_{s}$ is for nanosolid-particles, $\rho_{f}$ is for base fluid. 
Equations (3) and (4) admit self-similar solution of the appearance

$$
u=\alpha x f_{\eta}, \quad v=-\sqrt{\alpha v_{f}} f(\eta)
$$

where, subscript $\eta$ denotes the derivative.

In the stream function formulation Eq. (6), Eqs. (3) and (4) reduce to

$$
\frac{\partial^{3} \psi}{\partial y^{3}}+\frac{\rho_{n f}}{\mu_{n f}} \frac{\partial\left(\psi, \frac{\partial \psi}{\partial y}\right)}{\partial(x, y)}-\frac{\sigma B_{0}^{2}}{\mu_{n f}} \frac{\partial \psi}{\partial y}=0,
$$

where the second term in the above equation is the Jacobian. Substituting $\psi(x, y)=\lambda \sqrt{v_{f}} x f(\eta)$, into Eq. (8) and following ordinary differential equation

$$
f_{\eta \eta \eta}+(1-\phi)^{2.5}\left\{\left(1-\phi+\frac{\rho_{s}}{\rho_{f}} \phi\right)\left\{f f_{\eta \eta}-\left(f_{\eta}\right)^{2}\right\}-\frac{\sigma B_{0}^{2}}{\alpha \rho_{f}} f_{\eta}\right\}=0,
$$

This can be rewritten as,

$$
f_{\eta \eta \eta}+\Gamma_{1}\left\{\Gamma_{2}\left\{f f_{\eta \eta}-\left(f_{\eta}\right)^{2}\right\}-Q f_{\eta}\right\}=0,
$$

where, $\Gamma_{1}=(1-\phi)^{2.5}, \Gamma_{2}=1-\phi+\frac{\rho_{s}}{\rho_{f}} \phi$ and $Q=\frac{\sigma B_{0}^{2}}{\alpha \rho_{f}}$ is the Chandrasekhar number ( $\sqrt{Q}$ is called Hartmann number).

The associated boundary conditions are given by

$$
f(0)=V_{c}, \quad f_{n}(0)=\lambda, \quad f^{\prime}(\infty) \rightarrow 0,
$$

where, $V_{c}=\frac{v_{c}}{\sqrt{\alpha v_{f}}}$ suction/injection, $\lambda$ represents stretching/shrinking parameter, $\lambda>0$ represents stretching sheet, $\lambda<0$ represents shrinking sheet and $\lambda=0$ for fixed surface.

We search the solution of the laminar boundary value problem (9) and (10) in the following closed analytical form,

$$
f(\eta)=V_{C}+\lambda\left[\frac{1-\operatorname{Exp}(-\beta \eta)}{\beta}\right]
$$

where, $\beta>0$ must satisfy the quadratic equation,

$$
\begin{aligned}
& \zeta^{2}-\Gamma_{1} \Gamma_{2} V_{c} \zeta-\Gamma_{1}\left(\Gamma_{2} \lambda+Q\right)=0, \\
& \beta=\frac{\Gamma_{1} \Gamma_{2} V_{c} \pm \sqrt{\left(\Gamma_{1} \Gamma_{2} V_{c}\right)^{2}+4 \Gamma_{1}\left(\Gamma_{2} \lambda+Q\right)}}{2},
\end{aligned}
$$

The discriminant of Eq. (13) is positive when $\lambda \geq 0$ and it can be negative, if $\lambda<0$. In the latter case the discriminant is non-negative only if

$$
\lambda \geq \frac{\left(\Gamma_{1} \Gamma_{2} V_{c}\right)^{2}}{4}-\frac{Q}{\Gamma_{2}} .
$$

So in the case of a shrinking sheet if the inequality (13a) is not satisfied it is impossible to find $\beta$ and the analytical solution of the required form does not exist.

Suppose now that the discriminant is $\geq 0$ and distinguish some cases.

Case (i): If $V_{c}=0$ and $\Gamma_{2} \lambda+Q>0$, then $\beta=\Gamma_{1} \sqrt{\Gamma_{2} \lambda+Q}$, if $V_{c}=0$ and $\Gamma_{2} \lambda+Q \leq 0$ it is not possible to find $\beta$. 
Case (ii): If $V_{c} \neq 0$ and $\Gamma_{2} \lambda+Q>0$, then the quadratic equation admits two roots of different sign and

$$
\beta=\frac{\Gamma_{1} \Gamma_{2} V_{c} \pm \sqrt{\left(\Gamma_{1} \Gamma_{2} V_{c}\right)^{2}+4 \Gamma_{1}\left(\Gamma_{2} \lambda+Q\right)}}{2} .
$$

If $V_{c} \neq 0$ and $\Gamma_{2} \lambda+Q<0$, then the quadratic equation admits two roots of the same sign; if $V_{c}>0$ the two roots are positive and so $\beta$ has two possible values:

$$
\frac{\Gamma_{1} \Gamma_{2} V_{c}+\sqrt{\left(\Gamma_{1} \Gamma_{2} V_{c}\right)^{2}+4 \Gamma_{1}\left(\Gamma_{2} \lambda+Q\right)}}{2}
$$

and

$$
\frac{\Gamma_{1} \Gamma_{2} V_{c}-\sqrt{\left(\Gamma_{1} \Gamma_{2} V_{c}\right)^{2}+4 \Gamma_{1}\left(\Gamma_{2} \lambda+Q\right)}}{2} .
$$

Therefore in this case the problem (9) and (10) admits two analytical solutions. If $V_{c}<0$, the two roots are negative and so the problem does not admit a solution in closed form. If $\Gamma_{2} \lambda+Q=0$, and $V_{c}>0$, then $\beta=\Gamma_{1} \Gamma_{2} V_{c}$; if $\Gamma_{2} \lambda+Q=0$ and $V_{c}<0$, then it is impossible to find $\beta$.

Finally if $\lambda<0$, and the discriminant is equal to 0 , then in the case $V_{c}>0$ we have $\beta=\frac{\Gamma_{1} \Gamma_{2} V_{c}}{2}$, while in the case $V_{c}<0$ it is impossible to find $\beta$. When it is impossible to find $\beta$ one can try to solve the problem numerically. More over the possibility of two values of $\beta$ is not surprising because in the studies on the flows of the classical fluids with a stretching/shrinking sheet dual solutions have been found in the literature.

\section{Skin friction}

Wall shearing stress $\tau_{w}$ the expression is given by:

$$
\tau_{w}=-\mu_{n f}\left(\frac{\partial u}{\partial y}\right)_{y=0}=-\frac{1}{(1-\phi)^{2.5}} \rho_{f} \sqrt{v_{f} \alpha^{3}} x f_{\eta \eta}(0) .
$$

Substituting $u=\alpha x \lambda e^{-\beta \eta}$ in Eq. (14), we get

$$
\tau_{w}=\mu_{n f} \alpha^{3} x \beta \sqrt{\frac{\lambda}{v_{f}}} .
$$

\section{Results and discussion}

The present article is the generalization of the classical work of Crane (1970) flow and nanofluid driven by stretching/shrinking sheet with external magnetic field and suction. The classical Crane solution of the linear stretching sheet is extensive to include nanofluid, shrinking and suction/injection of weakly electrically conducting Newtonian fluids and also three types nanofluids, namely Copper $(\mathrm{Cu})$, alumina $\left(\mathrm{Al}_{2} \mathrm{O}_{3}\right)$ and Titania $\left(\mathrm{TiO}_{2}\right)$ in water as the base fluid. The basic boundary layer equation of momentum field is mapped into highly nonlinear ordinary differential equations via similarity transformations. Similarity solution is obtained for the velocity distribution. The velocities are decreasing function of $\eta$ as it is an exponential function with negative argument. It is 
apparent from Eq. (11), that is $\beta$, which is function of the suction/injection parameter $V_{c}$, with $V_{c}<0$ for suction, $V_{c}>0$ for injection and $V_{c}=0$ is the case when the surface is impermeable, stretching/shrinking parameter $\lambda, \lambda>0$ for stretching sheet, $\lambda<0$ for a shrinking and $\lambda=0$ for fixed surface and Chandrasekhar number $Q$, shows to the slope of above exponentially decreasing velocity profiles.

Figures 2, 3 and 4 reveals the influences of Chandrasekhar number $Q$, on the laminar boundary layer flow field. The presence of Chandrasekhar number $Q$ sets in Lorentz force effect, which consequences in the retarding effect on the velocity field. As the values of Chandrasekhar number $Q$, increase, the retarding force increases and consequently the velocity decreases. The same effect is observed for increasing values of $V_{c}>0$, it is also clear that increasing values of $Q$ results in flattening of $f_{\eta}$. These figures reveals that velocity profiles are going closer to the wall and the boundary layer thickness becomes thinner for the increasing $Q$. It is seen that the velocity is going closer to the wall and boundary layer thickness becomes thinner for larger $Q$. The reason behind this

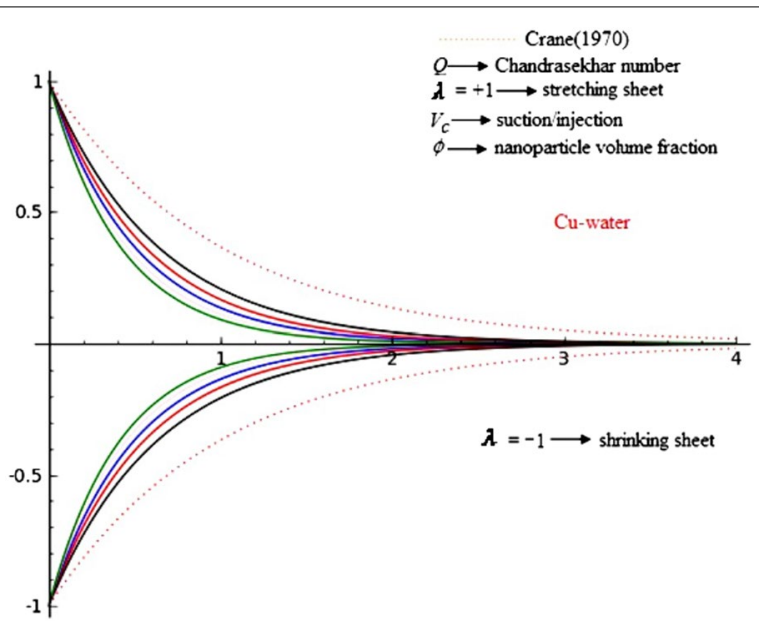

Fig. 2 Effects of Chandrasekhar number $Q$ on axial $f_{\eta}$ velocity in the case of Copper (Cu)-water with $\phi=0.2$ and $V_{c}=0.4$

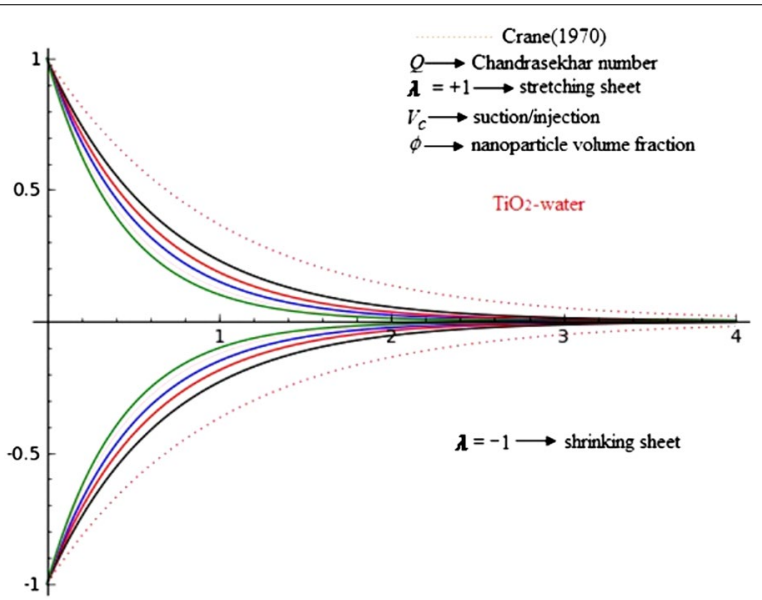

Fig. 3 Effects of Chandrasekhar number $Q$ on axial $f_{\eta}$ velocity in the case of Alumina $\left(\mathrm{Al}_{2} \mathrm{O}_{3}\right)$-water with $\phi=0.2$ and $V_{c}=0.4$ 


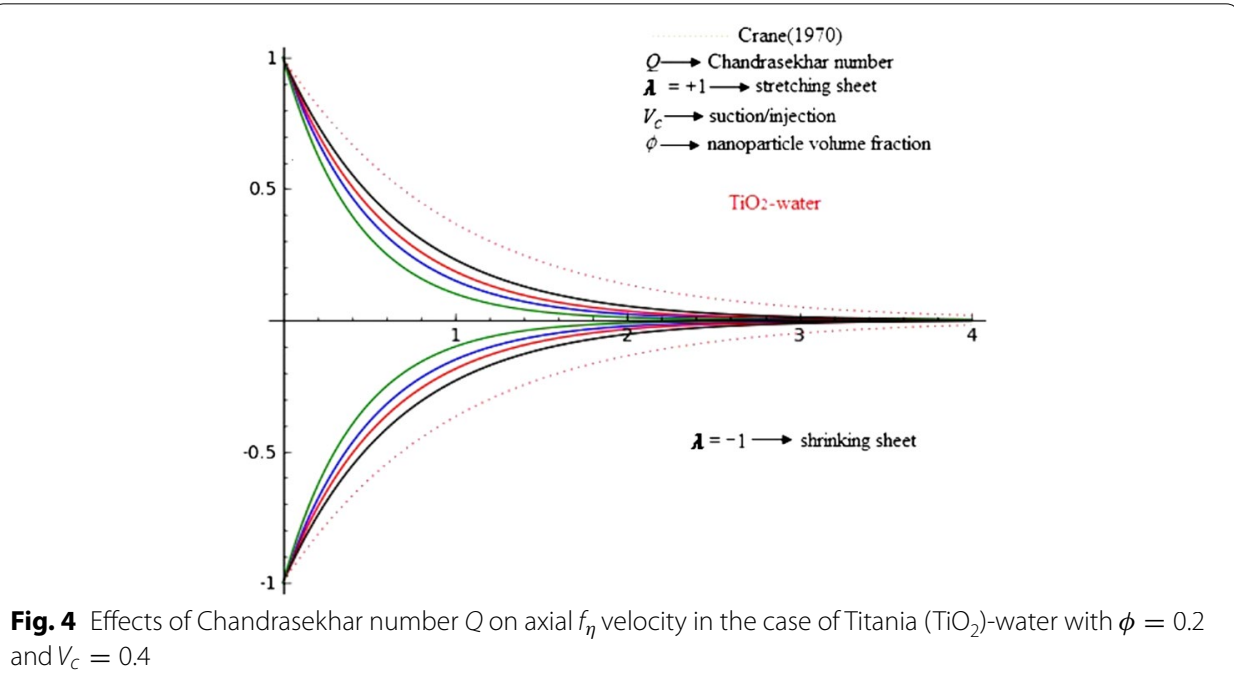

is that increase in $Q$ results the increase in Lorentz force which in turn produce more resistance to the velocity field. Physically, present phenomena occur when magnetic field can induced current in the conductive fluid, then it create a resistive-type force on the fluid in the boundary layer, which slow down the motion of the fluid. So finally, it is conclude that magnetic field is used to control boundary layer separation. The thickness of MHD boundary layer also depends upon the $\lambda$. For $\lambda=-1$, the laminar boundary layer thickness is larger than a $\lambda=+1$ and the effects of $Q$ are more pronounced. These effects are negligible for $\lambda=1$.

\section{Concluding remarks}

The laminar boundary layer flows in a nanofluid induced as a result of motion of a stretching/shrinking sheet has been presented. We study only analytical solution of the problem and some important results of the study are concluded as follows:

1. The axial velocity and transverse velocity, is a decreasing function of $\eta$ as it is an exponential function with negative argument.

2. Increasing values of the $Q$ results in pulling down of velocity profiles.

3. Velocity profiles decrease with an increase in $Q$ (Ferraro and Plumpton 1961) and (Borrelli et al. 2015).

4. The velocity components transverse velocity $f$ and axial $f_{\eta}$ are reveals for different values of the $Q$, the velocity decreases with increases in the $Q$ due to an increase in the Lorentz drag force that opposes the fluid motion.

5. The increase of $Q$ leads to the increase of skin friction parameter in all the cases of suction/injection.

6. The classical Crane (1970) flow is recovered from Eq. (13) for $V_{c}=Q=\phi=0$ and $\lambda=\Gamma_{1}=\Gamma_{2}=1$.

7. The classical Pavlov (1974) flow is recovered from Eq. (13) for $V_{c}=\phi=0$ and $\lambda=\Gamma_{1}=\Gamma_{2}=1$. 
8. The Gupta and Gupta (1977) flow is recovered from Eq. (13) for $\phi=0$ and $\lambda=\Gamma_{1}=\Gamma_{2}=1$.

9. The skin friction is lower for stretching and higher for shrinking sheets.

10. The effect of increasing the $V_{c}$ and the $Q$ is to increase the velocity and decrease the laminar boundary layer thickness in shrinking case (Borrelli et al. 2012, 2013a, b).

11. The heat transfer at the surface of the sheet increases with the increasing suction/ injection $V_{c}$ and the nanoparticle solid volume fraction $\phi$.

\section{List of symbols}

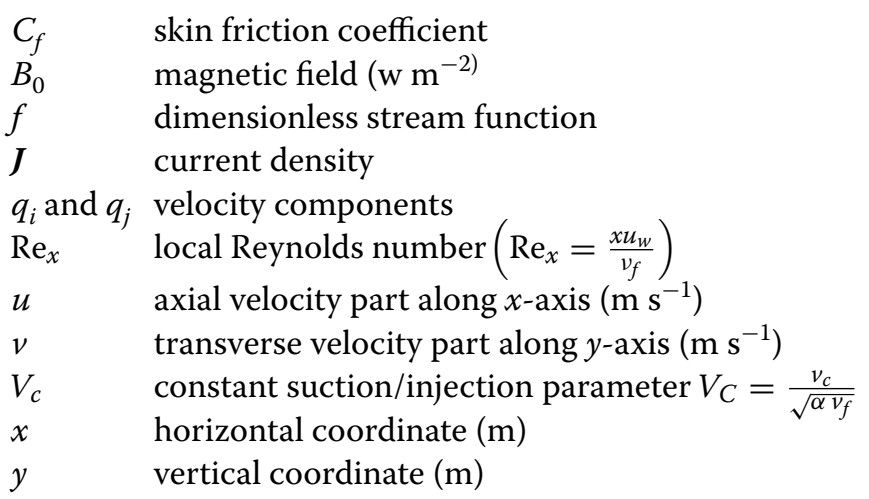

\section{Greek symbols}

$\alpha \quad$ constant in the sheet coefficient $\left(\mathrm{s}^{-1}\right),(\alpha>0)$

$\lambda \quad$ constant, represents stretching/shrinking parameter

$\eta \quad$ similarity variable $=\left(\sqrt{\frac{\alpha}{v_{f}}}\right) y$

$\mu_{n f} \quad$ viscosity of the nanofluid $\left(\mathrm{kg} \mathrm{m}^{-1} \mathrm{~s}^{-1}\right)$

$v_{f} \quad$ kinematic viscosity of the fluid $\left(\mathrm{m}^{2} \mathrm{~s}^{-1}\right)$

$\rho_{n f} \quad$ density of the nanofluid $\left(\mathrm{kg} \mathrm{m}^{-3}\right)$

$\rho_{f} \quad$ density of the fluid $\left(\mathrm{kg} \mathrm{m}^{-3}\right)$

$\rho_{s} \quad$ density of the nanosolid particles

$\sigma \quad$ electrical conductivity of fluid $\left(\mathrm{mho} \mathrm{m}^{-1}\right)$

$\tau_{w} \quad$ wall shearing stress $\left(\mathrm{m}^{2} \mathrm{~s}^{-1}\right)$

$\phi \quad$ nanoparticle volume fraction

$\psi \quad$ physical stream function $\left(\mathrm{m}^{2} \mathrm{~s}^{-1}\right)$

\section{Subscripts/superscripts}

$\begin{array}{ll}0 & \text { origin } \\ f & \text { fluid } \\ \mathrm{s} & \text { solid } \\ w & \text { wall condition } \\ \infty & \text { for from the sheet } \\ f_{\eta} & \text { first derivative w.r. t. } \eta \\ f_{\eta \eta} & \text { second derivative w.r. t. } \eta \\ f_{\eta \eta \eta} & \text { third derivative w.r. t. } \eta\end{array}$




\section{Authors' contributions}

The authors have contributed equally in conception and design of the study as well as analyzing and drafting of the manuscript. All authors read and approved the final manuscript.

\section{Author details}

${ }^{1}$ Department of Mathematics, Government First Grade College for Women, Hassan, Karnataka 573 201, India. ${ }^{2}$ Department of Mathematics, SHDD Government First Grade College, Paduvalahippe, Hassan, Karnataka 573 201, India. ${ }^{3}$ Department of Theoretical Mechanics, Tomsk State University, 36 Lenin Avenue, Tomsk 634050, Russia.

\section{Acknowledgements}

Author Dr. U. S. Mahabaleshwar (USM) thanks and wishes to Bharat Ratna Prof. Dr. C. N. R. Rao F.R.S. Honorable President VGST, Department of IT, BT Science and Technology, Bangalore, INDIA, for supporting this work under Seed Money to Young Scientists for Research (\# VGST/SMYSR/GRD-304/2013-14) and USM would like to acknowledge the receipt of Asia Bridge Fellowship, to thank to Professor Dr. Akira Nakayama, Shizuoka University, Japan for his hospitality.

\section{Competing interests}

The authors declare that they have no competing interests.

Received: 9 February 2016 Accepted: 20 October 2016

Published online: 02 November 2016

\section{References}

Andersson HI (1995) An exact solution of the Navier-Stokes equations for MHD flow. Acta Mech 113:241-244

Andersson HI (2002) Slip flow past a stretching surface. Acta Mech 158:121-125

Borrelli A, Giantesio G, Patria MC (2012) MHD oblique stagnation-point flow of a Newtonian fluid. ZAMP 63:271-294

Borrelli A, Giantesio G, Patria MC (2013a) On the numerical solutions of three-dimensional MHD stagnation-point flow of a Newtonian fluid. IJPAM 86:425-442

Borrelli A, Giantesio G, Patria MC (2013b) Numerical solutions of three-dimensional MHD stagnation-point flow of a micropolar fluid. Comput Math Appl 66:472-489

Borrelli A, Giantesio G, Patria MC (2015) Magnetoconvection of a micropolar fluid in a vertical channel. Int J Heat Mass Transf 80:614-625

Choi SUS (1995) Enhancing thermal conductivity of fluids with nanoparticles, vol 66. InternationalMechanical Engineering Congress and Exposition, San Francisco, pp 99-105

Choi SUS, Zhang ZG, Yu W, Lockwood FE, Grulke EA (2001) Anomalously thermal conductivity enhancement in nanotube suspensions. Appl Phys Lett 79:2252-2254

Crane LJ (1970) Flow past a stretching plate. ZAMP 21:645-647

Ferraro VCA, Plumpton C (1961) An introduction to magneto-fluid mechanics. Oxford University Press, London Fisher EG (1976) Extrusion of plastics, 3rd edn. Newnes-Butterworld, London

Gupta PS, Gupta AS (1977) Heat and mass transfer on a stretching sheet with suction or blowing. Can J Chem Eng 55:744-746 Keblinski P, Eastman JA, Cahill DG (2005) Nanofluids for thermal transport. Mater Today 8:36-44

Pavlov KB (1974) Magnetohydrodynamic flow of an incompressible viscous liquid caused by deformation of plane surface. Magnetnaya Gidrodin 4:146-147

Sakiadis BC (1961a) Boundary-layer behavior on continuous solid surfaces I: the boundary layer on a equations for two dimensional and axisymmetric flow. AlChE J 7:26-28

Sakiadis BC (1961b) Boundary-layer behavior on continuous solid surfaces II: the boundary layer on a continuous flat surface. AlChE J 7:221-225

Sakiadis BC (1961c) Boundary-layer behavior on continuous solid surfaces III: the boundary layer on a continuous cylindrical surface. AlChE J 7:467-472

Siddheshwar PG, Mahabaleshwar US (2005) Effects of radiation and heat source on MHD flow of a viscoelastic liquid and heat transfer over a stretching sheet. Int J Non Linear Mech 40:807-820

\section{Submit your manuscript to a SpringerOpen ${ }^{\circ}$ journal and benefit from:}

Convenient online submission

- Rigorous peer review

- Immediate publication on acceptance

- Open access: articles freely available online

- High visibility within the field

Retaining the copyright to your article

Submit your next manuscript at $>$ springeropen.com 